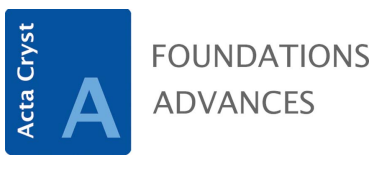

ISSN 2053-2733

\section{Introduction to Crystallography. By Frank Hoffmann. Springer, 2020. Hardback, pp. x+309. Price GBP 59.99. ISBN 9783030351090.}

\author{
Massimo Nespolo*
}

Université de Lorraine, CNRS, CRM2, Nancy, France. *Correspondence e-mail: massimo.nespolo@univ-lorraine.fr

Introduction to Crystallography by Frank Hoffmann is the English version of a German textbook (Hoffmann, 2016). It consists of nine chapters and three appendices, followed by a detailed index spanning three pages. About two-thirds of the content (the first six chapters) is devoted to crystal symmetry, and the other third to the description of some crystal structures, aperiodic crystals and some notions of reticular chemistry.

The original title (The Fascination of Crystals and Symmetry) is somewhat more loyal to the content of the book. Indeed, the more formal English title Introduction to Crystallography might suggest content in the lineage of classical textbooks like Buerger's (1956) book, for example. Instead, Hoffmann's textbook systematically avoids every possible obstacle for the student less inclined to abstract thinking and mathematical descriptions, giving instead priority to an intuitive pictorial approach. It can be described as a 'how to' manual for an end user with insufficient basic knowledge to pretend accessing a more formal description ('we do not want to deal with group theory here', p. 94). Students of the earth sciences seem to be the natural target, but those enrolled in chemistry will also most likely enjoy this book. Life scientists could also profit from the simplicity of the presentation, although they will miss examples close to their field. Students of physics, on the other hand, will clearly be highly dissatisfied by the complete lack of formal descriptions (no metric tensor, no matrices, no calculations at all).

Once we accept the absence of any formal approach to the subject, the book stands out from many concurrent, even much more pretentious, works for its rigour of language (with some exceptions discussed below) which, although unable to reach perfection, is certainly higher than that in most of the books published in recent years. Moreover, the book comes with an online resource depository companion, where the reader finds paper models for the 32 (geometric) crystal classes, short videos showing animations of the actions of glide planes and screw axes whose static versions are published in the book, and CIF files of crystal structures presented in the text.

Chapter 1 (32 pages) is humbly entitled Introduction. Contrary to so many textbooks that present a dry and unappetizing approach to the basic definitions, it provides an unusual but tasty 'first bite' of the subject. It starts with a comparison of the sizes of objects differing by powers of 10 (reference to the well known short movie acknowledged) to introduce the notion of crystal structure in a very intuitive way. This is followed by a crystallographic poem summarizing some crystallographic terms that might sound cryptic to the beginner, and a graphic backbone using the words 'crystal' and 'lattice' taken from the well known book by the Canadian poet Christian Bök (2003). At this point, the reader is, one hopes, hooked by the fascinating beginning of the narration, which goes on to emphasize the difference between a structure and its lattice, through examples of periodic (as well as some counter-examples of non-periodic) arrangements of objects (tuna tins, bags of crisps, cola cans). Knowing how severe is the confusion between structure and lattice even in the mind of confirmed scientists (Nespolo, 2019), the efforts made to prevent any possible such confusion in the mind of the new reader is extremely welcome. The second part of the chapter goes back to a more traditional description and introduces the notions of unit cell and crystal system. Most welcome is the emphasis put on the restrictions imposed on the cell parameters by symmetry and the incorrect way most textbooks present them (Tables 1.1 and 1.2), something I have long been pestering about without much success (Nespolo, 2015). The three examples on 
pages 26-27 point out how, from the cell parameters, one can exclude higher symmetries, but not lower ones, as happens in the case of metric specialization, a phenomenon not sufficiently well known (de Gelder \& Janner, 2005a,b). Example 2 is however incomplete, because from the cell parameters $a=b=c, \alpha=\beta=\gamma=90^{\circ}$ the possibility of a trigonal structure is excluded, whereas in fact the cell parameters may correspond to those of a rhombohedral crystal with metric specialization of the interaxial angle. Also, Example 3 discusses cell parameters corresponding to a hexagonal unit cell and a monoclinic structure is excluded, which is incorrect because the unique monoclinic axis could have been taken along $\mathbf{c}$.

Chapter 2 (57 pages) deals with Crystal Shapes and Bravais Lattices. The subject is approached by discussing the morphology of crystals in some detail, as is traditionally done in mineralogical texts but sadly too often forgotten by modern textbooks. The approach remains essentially intuitive and the notions of form and zone are not even introduced. This is followed by a graphical explanation of the law of constancy of interfacial angles and the introduction of Miller indices, with many polychromatic figures that will certainly help the beginner to get used to these indices. Unfortunately, the common mistake of restricting Miller indices to coprime integers (which is untrue if the unit cell is not primitive; Nespolo, 2015) is present also in this book. Page 44 explains the difference between Miller and Laue indices, something too often neglected in textbooks. Unfortunately, Laue indices are written within parentheses, which should really only be used for Miller indices. The second part of the chapter is devoted to a discussion of primitive and centred unit cells and the enumeration of the (types of!) Bravais lattices. A glimpse of a change of reference system (change of unit cell) is provided through a two-dimensional example but no matrix treatment is offered. It is followed by a short discussion of atomic fractional coordinates and CIF files, and a tutorial for the VESTA software (Momma \& Izumi, 2011). Ten pages with many screenshots are devoted to explaining how to use the software; one might wonder if a textbook is the right place for this, although the end user will certainly appreciate it. The last part of the chapter is dedicated to a lengthy explanation of the differences between hexagonal, trigonal and rhombohedral crystals, something that is elementary but which nevertheless still gives headaches to even expert researchers. The case of a honeycomb is chosen to emphasize once more the difference between a structure and its lattice: anyone who does a quick internet search for the non-existent 'honeycomb lattice' and gets frightened at discovering the huge number of entries will certainly agree that the choice of this example is very wise.

Chapter 3 (35 pages) brings the title Symmetry (Is Everywhere) and is actually restricted to point symmetry. A more precise distinction between symmetry operations and symmetry elements would have been necessary (identity is not and does not have a symmetry element!). The first part of the chapter explains crystallographic point-symmetry operations, while the second part introduces (geometric) crystal classes.
Several drawings and photos make the presentation easy to follow. No difference is made between a crystallographic (type of) point group and the corresponding (geometric) crystal class. Unfortunately, some inconsistencies affect the presentation, like considering $[\overline{u v} 0]$ and $[u v 0]$ as two separate directions (page 113), or stating that 'All symmetry elements are shown in Fig. 3.33', while only those corresponding to the short Hermann-Mauguin symbol are actually drawn. Moreover, in Table 3.3 the 'long' (should be 'full') and 'short' Hermann-Mauguin symbols for trigonal groups are given with and without, respectively, the ' 1 ' symbol for the direction of missing symmetry, while its presence or absence in fact depends on the type of lattice (hexagonal or rhombohedral). The chapter ends with another tutorial on VESTA, on how to draw crystal morphologies.

Chapter 4 (24 pages), under the long title Symmetry in the Plane: About Wallpaper Patterns, Islamic Mosaics, Drawings from Escher, and Heterogeneous Catalysts, aims to provide a short introduction to two-dimensional patterns, with a brief excursus into the three-dimensional world. Several nice polychromatic figures make the reading pleasant. Unfortunately, we read about glide planes and rotation axes in two dimensions (where only glide lines and rotation points exist). As anticipated by the title of the chapter, plane groups are exemplified through ornaments and a couple of drawings taken from Escher's universally known work. The analysis then moves on to the role of surfaces in catalysis and, quite surprisingly, to examples of metals having simple structures (why here rather than in the following chapter?).

Chapter 5 (32 pages) brings the title Translational Symmetry Elements in Crystals and Space Groups I and does what the title says: it explains glide planes and screw axes and introduces the notion of space group, although without any element of group theory. Symmetry elements and symmetry operations are sometimes mixed up and this makes the reading somewhat disturbing. The choice of an achiral object (a locomotive) to show the effect of symmetry operations prevents the reader from understanding by simple inspection the difference between handedness-preserving (first kind) and handedness-reversing (second kind) operations. The space devoted to showing the difference between glide planes of types $a, b$ and $c$ could have been reduced by explaining that one just has to change the label of the axis along which the glide component is active. Several examples of objects presenting screw-rotational symmetry are shown, through photographs taken from nature, man-made objects and crystal structures. The second part of the chapter is a tutorial on how to read the Hermann-Mauguin symbols of space groups, with a few examples of geometric solids building up simple crystal patterns.

Chapter 6 (40 pages) deals with Space Groups II: International Tables and is essentially a tutorial on how to read off information from Vol. A of International Tables for Crystallography. Unfortunately, reference is still made to the 5th edition, while the 6th edition was published in 2016, and some statements in this chapter are therefore outdated. One may wonder if it is reasonable to devote 11 pages to listing the 
230 types of space group. Once again many diagrams and drawings make the text easy to follow, although the treatment remains intuitive and without any formalism. This does not prevent the author from teasing the reader when writing (page 208), 'you already know almost everything about crystallographic point and space groups': is it really necessary to flatter the reader in such an exaggerated way? A huge mistake occurs on pages 202-203, where, among the settings of spacegroup type No. 14, we find - twice! - $P 112_{1} / c$ instead of $P 112_{1} / b$. It should be obvious that a $c$-glide cannot occur perpendicular to the $c$ axis!

Chapter 7 (33 pages) deals with Some Real Crystal Structures: From Theory to Practice. The first part of the chapter is a tutorial on the use of the Mercury software, followed by some short notions about polymorphism and examples of very simple structures (rock salt, graphite, diamond). The last part of the chapter is an introduction to ferroelectricity, written in an intuitive and easy to follow way. Highly regrettable is the confusion about lattice and structure in the description of rock salt (page 236), and quite surprising, considering the care the author has put into making clear the difference between these two concepts.

Chapter 8 (17 pages) presents Forbidden Symmetry and discusses aperiodic crystals, as usual in a very intuitive way. One may wonder why a drawing that is clearly threedimensional (Fig. 8.15b) is described as 'two-dimensional'. Perhaps this is due to the common confusion between dimensionality and periodicity (Nespolo, 2019)?

The last chapter (25 pages) discusses Porous Crystals, Crystal Structures as Networks, and an Insight into Crystallographic Topology. It is essentially a brief introduction to reticular chemistry, which is sadly unknown among many crystallographers. After a short presentation of zeolites and metal-organic frameworks (MOFs), some notions of graph theory and topology are presented, which seem severely unbalanced with respect to the non-mathematical approach of the previous chapters. One may reasonably wonder how an audience considered reluctant to read about some basic notions of group theory will react to vertex symbols, genus, topological density and transitivity.

Three very short appendices provide answers to some exercises, a list of the minerals and compounds whose CIF files are available on the companion website, and a list of the resources available there.

The choice of keeping a very intuitive and graphical approach, almost empty of any formal treatment, has the advantage of avoiding scaring the less mathematically inclined reader. However, it also has obvious limitations: many concepts simply cannot be explained without some algebra. Moreover, despite the care taken over the rigorous presentation of each concept that is introduced and explained, some approximations and incorrect expressions have to be remarked on, which one hopes may be amended in a future revised edition.

(i) The explanation of why (geometric) crystal classes $\overline{6}$ and $\overline{6} m 2$ are placed in the hexagonal crystal system rather than the trigonal one is far from convincing. It would have been enough to explain that the horizontal mirror plane is incompatible with a rhombohedral lattice, which rules out the possibility of placing the crystal structure in the trigonal system. This simple argument is perhaps considered too abstract for the target readership?

(ii) A serious drawback which affects most chapters is the lack of distinction between a group (an element, an operation) and a type of group (type of element, type of operation). For example, we read on page 22 that 'the unit cell contains all symmetry elements of the crystal', which is clearly meaningless, the unit cell being finite but the crystal structure infinite (well, ignoring the surface of course). In the same way, the following page states that by translating the unit cell, 'no additional symmetry element can appear', while of course infinitely many new symmetry elements arise, although all of the same type as those spanning the unit cell.

(iii) Several examples are shown in two-dimensional space, for evident didactic reasons. Nevertheless, the Miller indices and direction indices are given with three digits, which is totally inconsistent. A statement (page 135) tries, and fails, to justify this inconsistent use across spaces of different dimension 'for the sake of a uniform presentation'. This needs to be fixed in the next edition.

(iv) The footnote on page 25 discusses the case of "no degrees of freedom' for cell parameters, whereas at least one degree of freedom is always present, even for cubic crystals (the length of the cell parameter).

(v) The inversion centre is listed among the symmetry elements of two-dimensional objects (page 99), but it cannot exist in even-dimensional spaces. On the following page it is stated that for two-dimensional objects it 'is identical to the presence of a twofold rotation axis'. Although one understands what the author means, this statement is doubly wrong: firstly because in a two-dimensional space there are no rotation axes but only rotation points; and secondly because a handedness-reversing operation (inversion) cannot be equated to a handedness-preserving operation (rotation), even though the effects of the two operations can be indistinguishable when applied to an achiral object. Here we face the limits of an informal pictorial description which carefully avoids any formalism.

(vi) Sublattices are incorrectly called 'superlattices' (page 271), an unfortunately common mistake which has its origin in the fact that the unit cell of a sublattice is a supercell and vice versa. A bit of group theory would have avoided this trap but, as we have seen, group theory has no right of citizenship in this book.

The technical editing has been done very carefully, as shown by the very few typographic errors remaining.

Overall, Hoffmann's book is clearly of little use for students of physics, but it is a nice introduction to the subject for students of the earth sciences, chemistry and possibly the life sciences. The number of subjects that are left out is too long to be listed exhaustively. Some (reciprocal space, diffraction or other methods of structure determination) are already covered by so many textbooks that the reader will have no 


\section{book reviews}

difficulty in finding the necessary resources. Others (crystallographic calculations, group theory) are dealt with in the wider literature in such a difference of depth and approach that some references suitable for the target readership would have been welcome. Nevertheless, considering that for an increasing fraction of students a formal and rigorous treatment has become highly repulsive, a textbook like the present one does have a role to play. It is, however, necessary to correct the mistakes and inconsistencies that I have pointed out, although these are not large in number. When preparing a second edition, the author may also wish to consider that the alternate use of 'lattice parameters', 'lattice constants' and 'cell parameters' may disconcert the reader. Choosing a unique term would be wiser ('cell parameters' being certainly the most suitable one, since the cell choice is not unique, whereas the lattice is uniquely defined).

\section{References}

Bök, C. (2003). Crystallography, 2nd revised ed. Toronto: Coach House Press.

Buerger, M. J. (1956). Elementary Crystallography. New York, London: John Wiley \& Sons, Inc./Chapman \& Hall.

Gelder, R. de \& Janner, A. (2005a). Acta Cryst. B61, 287-295.

Gelder, R. de \& Janner, A. (2005b). Acta Cryst. B61, 296-303.

Hoffmann, F. (2016). Faszination Kristalle und Symetrie. Cham: Springer.

Momma, K. \& Izumi, F. (2011). J. Appl. Cryst. 44, 1272-1276.

Nespolo, M. (2015). J. Appl. Cryst. 48, 1290-1298.

Nespolo, M. (2019). J. Appl. Cryst. 52, 451-456. 